\title{
Radiation Oncology Practices Owned by Private Companies? Does Corporate Medicine Work in Radiation Oncology?
}

\section{John Leung*}

Adelaide Radiotherapy Centre, 352 South Terrace, Adelaide, South Australia

\author{
Abstract \\ - Radiation oncology practised in public hospitals or private practice. \\ - Adelaide Radiotherapy Centre is sole private practice provider of services in South Australia. \\ - Corporate medicine not involved in radiation oncology. \\ - $\quad$ Adelaide Radiotherapy Centre bought out by private company in 2008. \\ - Rationale, benefits and disadvantages discussed.
}

Keywords: Radiation oncology practices; Corporate medicine; Private practice

\section{Introduction}

Corporate medicine has been an integral part of a number of branches of medicine for many years. There is however little data on radiation oncology in Australia.

Adelaide Radiotherapy Centre is the private practice provider of radiation oncology services in South Australia. It was founded in 1985 by two radiation oncologists. There were two doctors, one cobalt machine and less than fifteen staff at that time. In 2012, Adelaide Radiotherapy has eight radiation oncologists, one trainee and over 100 staff. The practice has three sites, St Andrews Hospital, Flinders Private Hospital and the Tennyson Centre. It has six linear accelerators, offers brachytherapy services, does IMRT and is involved in training of advanced trainees amongst other activities.

In 2002, Adelaide Radiotherapy Centre was approached by IMed Services for a buyout. IMed Services was acquiring radiology companies and was also interested in branching out into radiation oncology. There were four partners in the practice at the time and ultimately the deal was rejected because two partners rejected the offer. Subsequent to this, Adelaide Radiotherapy Centre decided to have a more formal board. It appointed an external chairman, who had a business and advisory role, but who was not a medical practitioner. It also appointed a Chief Executive Officer who had been a finance chief at various hospitals.

In 2007, a decision was made to sell the practice. Price Waterhouse Coopers aided in the tender process. There were at least five interested parties.

IMed Services (now owned by CVC Asia Pacific), successfully purchased the practice. The buy out was partial with the five doctor partners retaining ownership of a substantial part of the practice. IMed Services already owned one other radiation oncology practice in Australia - Radiation Oncology Associates - a practice based in New South Wales.

In September 2009, IMed (CVC Asia Pacific) sold off the radiation oncology and cardiology parts of the organisation to the Advent Group.

\section{Rationale}

\section{Help with expansion}

In 2007, Adelaide Radiotherapy Centre had two centres at South
Terrace and Flinders Private Hospital. The practice was keen to have two further sites developed. There was some trepidation amongst some of the doctors that the practice would not have the ability to deal with two major projects. However, this was not unanimous and certainly two out of the five doctors thought the practice would cope. However, the independent Chairman and the Chief Executive Officer thought that Adelaide Radiotherapy Centre may need external help. These two individuals have always been perceived as relatively neutral and having business expertise. When both of them were keen to use an external company to aid the practice, much more notice was taken.

\section{Business expertise}

Adelaide Radiotherapy Centre is unique in that there have been people with business background in the practice. As mentioned previously, the independent Chairman and the Chief Executive Officer have strong financial and business backgrounds. Some other practices in Australia still rely on doctors managing the business side of the practice. This is not necessarily beneficial as radiation oncologists do not necessarily have the knowledge to run a successful business, although they may be competent radiation oncologists. Private practices require not only good patient management, but sound business practice.

However, even with business focused individuals, it was thought more expertise could be added with a large company supporting the practice.

\section{Economic power}

A larger organisation with two different radiation oncology practices in Sydney and Adelaide was perceived to have improved leverage in contract negotiations. Linear accelerators, planning systems, brachytherapy equipment could all come at substantially

*Corresponding author: John Leung, Adelaide Radiotherapy Centre, 352 South Terrace, Adelaide, South Australia, Tel: +61 88228 6700; Fax +61 88232 1243; E-mail: john.leung@adradcentre.com.au

Received June 12, 2012; Accepted June 21, 2012; Published June 25, 2012

Citation: Leung J (2012) Radiation Oncology Practices Owned by Private Companies? Does Corporate Medicine Work in Radiation Oncology? J Nucl Med Radiat Ther S6:008. doi:10.4172/2155-9619.S6-008

Copyright: (c) 2012 Leung J. This is an open-access article distributed under the terms of the Creative Commons Attribution License, which permits unrestricted use, distribution, and reproduction in any medium, provided the original author and source are credited. 
discounted prices if bought in bulk. This would be further enhanced if more practices were acquired.

\section{Formation of a national radiation oncology network and new business}

IMed made it very clear that acquiring Adelaide Radiotherapy Centre was part of a process to form a national network. They were involved or about to be involved in discussions with other practices. If a national network could be formed, this would give the group strong leverage not just in contract negotiations for equipment, but could provide many other potential benefits. Sharing of staff, uniform treatment protocols, enhanced quality of care and political leverage might all be possible.

\section{Benefits}

\section{Maintenance of clinical dependence}

It was made clear at the outset that there would be no interference with clinical management. A fear by the radiation oncologists was that profitability would interfere with clinical management. Selection of patients for radiation, planning, dose fractionation schedules and patient care have remained in the jurisdiction of the doctors only. A clinical management committee which comprised the Adelaide Radiotherapy Centre doctors, a radiation oncology associate doctor and a member of the IMed board was set up. This practice committee has made the clinical decisions based on optimal patient care.

\section{Removal of financial and business responsibility}

Personal guarantees for bank loans on equipment, facilitating etc. have all been removed from the doctors. Personal guarantees have concerned some, but not all the doctors. However, there is little financial risk for individual practitioners now. The business part of the practice has largely been taken over by the new company.

\section{Economic benefits}

The radiation oncologist partners received a cash windfall and shares from the partial buy out. However, as the practice would be partially owned by a private company, each doctor's future income would be less than before. This makes sense as the new company would now keep some of the profits for themselves as they owned part of the practice.

The traditional thinking is that older partners who are about to retire would benefit much more than younger partners. However, the younger doctors have many years of future earnings to compensate them. Adelaide Radiotherapy Centre asked Price Waterhouse Coopers to analyse this in more detail. The overall conclusion was that a partial sell out would benefit all partners even in the long term.

\section{Succession planning}

The traditional method of joining a private practice is to work as an associate and then be invited to be a partner. If the practice is partially owned by another company, this might no longer be valid. Future radiation oncologists would have little incentive to join. However, the new company was able to formulate a plan where future radiation oncologists would be able to purchase shares and become partners in a much larger company. This plan has been officially integrated into the practice. The practice has attracted two new associates.

\section{Stable workload}

An unexpected occurrence has been an actual decrease in workload for three of the five partner radiation oncologists. One other has had an increased workload and the other has remained the same. This is despite the overall group seeing more patients. This has happened primarily because of the addition of the new radiation oncologists. It was originally anticipated that the doctors would have to work much harder as the emphasis would be on profits.

\section{Increased structure and business expertise}

Adelaide Radiotherapy Centre has always had reasonable structure with a partners' meeting monthly and various partners assigned to look after the business, clinical, technical and quality control parts of the practice.

However, this has become much more formalised with minutes taken, accountability and follow up of issues discussed. Issues are not left unresolved, but are discussed with a resolution.

There is now an overall board, a local management board and several committees.

\section{Approachability of new company}

A pleasant surprise has been approachability of the key players in the new company. They have been pleasant, cooperative and effective in their dealings so far. This was especially important in the initial stages of the transaction and for the first year afterwards when some of the radiation oncologists were very anxious.

\section{Leverage in purchasing equipment}

The new company has been able to purchase a new CMS planning system, CT scanners, linear accelerators and other equipment at substantial discounts. Whether Adelaide Radiotherapy Centre as a sole company would have been able to purchase the equipment at the same prices is unknown.

\section{Disadvantages}

\section{Loss of control}

One of the major benefits of traditional radiation oncology private practice is that partners control everything and they choose working hours, clinics, areas of interest, holidays, staff and dominate all facets of the practice. Basically, they can do anything they like within reason. This may occur in public hospitals, but is accentuated in private practice.

Although radiation oncologists still retain clinical independence, they are now accountable to another body and do not always get exactly what they want. Two interesting examples of this were the choice of a new planning system and holiday times. The majority of the doctors were happy to retain the Pinnacle Planning system, but were outvoted to obtain the CMS Focal Planning system. CMS Focal was cheaper and being used by radiation oncology associates in Sydney. Holiday leave is another interesting example. In the past, radiation oncologists chose exactly when they wanted to take leave. Unfortunately, sometimes this resulted in three or four people away at the same time. Nowadays, application forms are submitted to ensure smooth operation of the practice.

Loss of total control was always anticipated though and has to be accepted when another company partially owns the practice.

\section{Focus on financial and statistical data}

A successful radiation oncology private practice must focus 
on the business as well as the clinical side of the practice. Adelaide Radiotherapy Centre has done this more than adequately with certain partners taking active interest, the employment of individuals with financial backgrounds, monthly reviews and regular monitoring by the accountants.

When IMed took over, a concern that too much emphasis has been placed on this. An example was when practice income temporarily dropped below budget in 2008 to 2009. In the past, partners would have expressed concern and tried to resolve this, but realised income and budgets were not the only concern. However, the non doctor partners called so many meetings to seek explanations and remedial action that the radiation oncologists felt uncomfortable. The practice is currently meeting budget.

Monthly meetings with financial reports are so comprehensive now (up to thirty pages) that even the more financially minded partners have trouble following them.

\section{Meetings}

This has diminished recently, but the first year involved so many meetings that it became intrusive. There were board, practice management and other special meetings often convened at short notice. This in part was due to the new company trying to understand the structure and running of Adelaide Radiotherapy Centre.

\section{Global financial crisis impact}

Adelaide Radiotherapy Centre had lines of credit and loan facilities with the major banks. When IMed (owned by CVC Asia Pacific) bought part of the company, these facilities were all abolished. IMed had their own structures. However, when the global financial crisis came, the major banks refused to lend and credit became very tight. The net result was that Adelaide Radiotherapy Centre paid for equipment and expansion directly out of cash flow instead of loans. A shortage of actual cash meant that although profits were excellent on paper, they could never be fully realised because there was no actual cash.

This specific problem eventually led to IMed (CVC Asia Pacific) divesting itself from radiation oncology and cardiology in 2009 .The radiation oncology and cardiology practices formed their own company.

\section{Impact on non radiation oncology staff}

This is probably unknown at present and may require a questionnaire or survey. There is anecdotal evidence that radiation therapists and administrative staff are more stressed because of increased workload. However, whether this is due to other factors such as a shortage of radiation therapists is unknown.

\section{Conclusion}

Does radiation oncology and corporate medicine mix? The answer depends on who you are. If you are seeking a fresh challenge, need extra business expertise, expanding beyond your means or seeking a business opportunity, and then the answer is yes. It is also yes if you are retiring in five to ten years or if you are a radiation oncologist interested in clinical work only requiring others to run the business side.

However, if you wish to remain in total control without outside influence and have confidence in your own business abilities, then the answer is no. It is also negative if you believe your practice can solely handle expansion plans, and you wish to remain in much defined boundaries within your state.

Which one are you? 